\title{
Bone Scintigraphy SPECT/CT Evaluation of Mandibular Condylar Hyperplasia
}

\author{
Zhiyun Yang, Tameron Reed, and Becky H. Longino \\ Department of Radiology, Louisiana State University Health Sciences Center/University Health, Shreveport, Louisiana
}

\begin{abstract}
Mandibular condylar hyperplasia $(\mathrm{CH})$ is a complex developmental deformity resulting in asymmetries of the hyperplastic condyle. Bone scan SPECT is a sensitive and accurate method of detecting the growth activity of this disorder. This method can be used to quantitate the radionuclide uptake differences between the left and right condyles. Uptake differences of $10 \%$ or more between the left and right condyles, with increased uptake ipsilateral to the $\mathrm{CH}$, are considered to be evidence of active growing $\mathrm{CH}$. Quantitative assessment of $\mathrm{CH}$ is important to select an appropriate treatment course. Degenerative arthropathies of the temporomandibular joints may result in altered uptake, but this is mostly associated with the side contralateral to the $\mathrm{CH}$. The CT portion of SPECT/CT is useful to assess the condylar dimensions and underlying bony changes.

Key Words: bone; SPECT; SPECT/CT; bone scintigraphy; condylar hyperplasia; mandibular condyle hyperplasia; temporomandibular disorders
\end{abstract}

J Nucl Med Technol 2016; 44:49-51

DOI: 10.2967/jnmt.115.158691

C ondylar hyperplasia $(\mathrm{CH})$ is a complex developmental deformity leading to asymmetries of the hyperplastic condyle. It causes facial asymmetry and malocclusion and can be associated with pain and dysfunction. Accurate assessment of condylar growth and establishment of disease activity provide guidance for treatment. Bone scan SPECT has demonstrated a performance in this application superior to planar imaging through the accurate and quantifiable assessment of mandibular condylar growth (1-3). The CT portion of SPECT/CT provides detailed anatomic information associated with growth activity.

\section{CASE REPORT}

A 40-y-old woman (who gave consent for study inclusion) presented with a 20 -y history of pain in the bilateral TMJs (temporomandibular joints), with pain worse

Received Apr. 8, 2015; revision accepted Jun. 2, 2015.

For correspondence or reprints contact: Zhiyun Yang, Department of Radiology, LSU Health Sciences Center/University Health, 1501 Kings Highway, P.O. Box 33932, Shreveport, LA 71130.

E-mail: zyang@|suhsc.edu

Published online Jun. 25, 2015.

COPYRIGHT (c) 2016 by the Society of Nuclear Medicine and Molecular Imaging, Inc. in severity on the left side. Bone scintigraphy was ordered to evaluate growth activity in the left $\mathrm{CH}$.

A limited examination of the TMJs consisted of radionuclide angiography and immediate postinjection images with 3-h delayed planar image collection after intravenous administration of $740 \mathrm{MBq}(20 \mathrm{mCi})$ of ${ }^{99 \mathrm{~m}} \mathrm{Tc}-$ methylene diphosphonate (MDP). Subsequent SPECT/CT was performed using a GE-640 SPECT/CT camera (GE Healthcare).

\section{DISCUSSION}

The CT 3-dimensional volume reconstruction image showed the patient's chin tilted to the right (Fig. 1), consistent with the patient's facial asymmetry on physical exam.

The SPECT results showed increased ${ }^{99 \mathrm{~m}} \mathrm{Tc}-\mathrm{MDP}$ uptake in the mandibular condyles compared with that of the clivus. Additionally, asymmetric MDP uptake was observed, with the right increased over the left side (Figs. 2 and 3). Calculated uptake was 55\% on the right (right condyle counts divided by the sum of right and left condyle counts) and $45 \%$ on the left. The $10 \%$ difference observed with increased uptake on the side contralateral to the $\mathrm{CH}$ indicated inactive growth. This asymmetric 99m Tc-MDP uptake was not observed in the planar images (not shown).

The low-dose CT showed that the left mandibular condyle was larger $(21.4 \times 22.5 \times 12.2 \mathrm{~mm})$ than the right mandibular condyle $(17.8 \times 16.1 \times 9.8 \mathrm{~mm})$ in the axial, coronal,

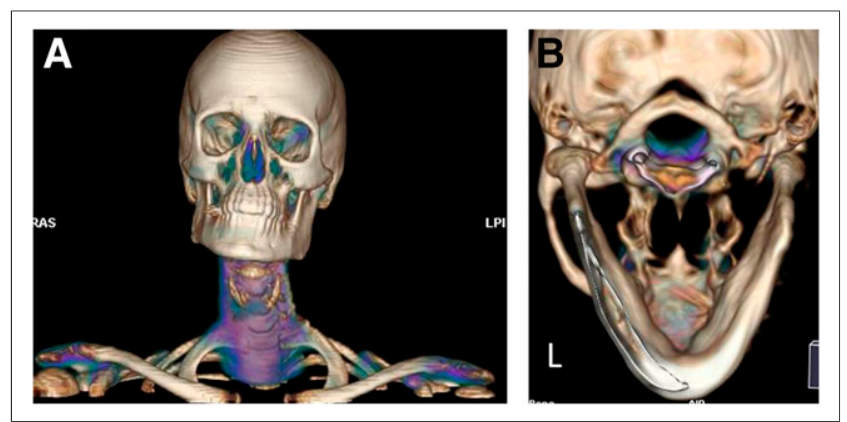

FIGURE 1. CT 3-dimensional volume reconstruction image shows asymmetry of mandible. (A) Right mandibular ramus is shorter vertically than left mandibular ramus. Corresponding posterior volume reconstruction views (B) further demonstrate larger left condyle. These findings are consistent with patient's facial asymmetry as found on physical exam. 


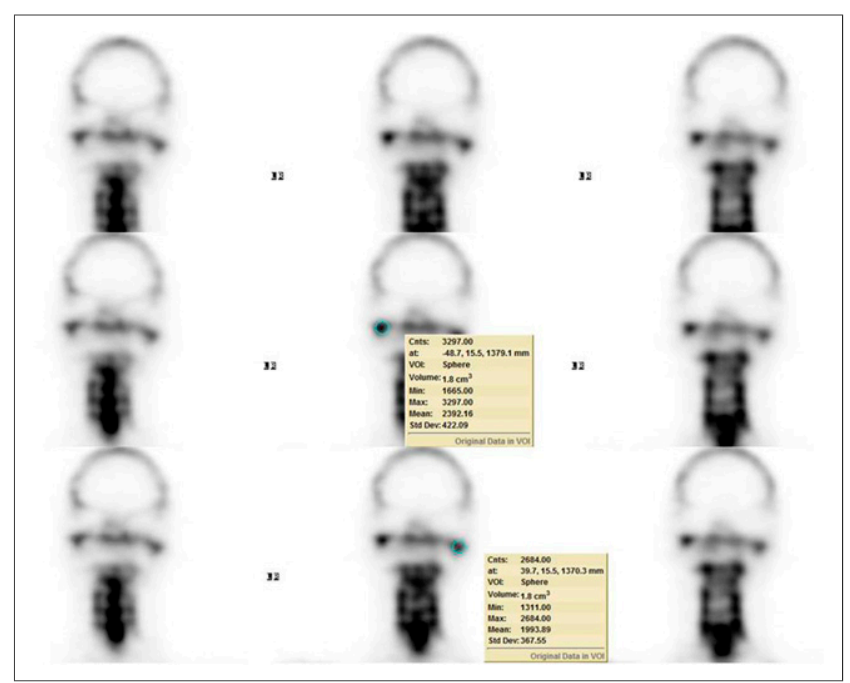

FIGURE 2. Same serial coronal SPECT images are displayed with measured counts shown on rows 2 and 3 . Volumetric 3dimensional quantification software (GE Healthcare) was used to indicate region of interest on each condyle. Total counts of right condyle, $3,297.00$ counts $/ 1.8 \mathrm{~cm}^{3}$, and of left condyle, $2,684.00$ counts $/ 1.8 \mathrm{~cm}^{3}$, are shown on rows 2 and 3 , respectively. There is increased ${ }^{99 m T c-M D P}$ uptake in mandibular condyles compared with that of clivus. SPECT scan was obtained using the optimal 640 hybrid camera (GE Healthcare) with dual heads and a pair of low-energy, high-resolution collimators. SPECT data were acquired from 120 projections over $360^{\circ}$ at $30 \mathrm{~s}$ per projection on a matrix of $128 \times 128$. Three-dimensional SPECT images were reconstructed on Xeleris workstation with Volumetrix MI Evolution software (GE Healthcare) for bone using following parameters: 2 iterations, 10 subsets, Butterworth filter with cutoff frequency at 0.48 , and power at 10 . Slice thickness was $4.42 \mathrm{~mm}$. Cnts = counts; Max = maximum; Min = minimum; Std Dev = standard deviation; $\mathrm{VOI}=$ volume of interest.

and sagittal reconstruction images, respectively (Fig. 4). The TMJ spaces were narrowed with sclerosis (particularly on the right side) (Fig. 5). These findings led to 2 conclusions: the left mandibular condyle was larger with less ${ }^{99 \mathrm{~m}} \mathrm{Tc}-\mathrm{MDP}$ uptake, indicating an inactive left mandibular $\mathrm{CH}$; and the TMJs, narrowed with sclerosis, had increased ${ }^{99} \mathrm{~m} \mathrm{Tc}-\mathrm{MDP}$ uptake, indicating degenerative changes, with the right side being more advanced.

Patients with $\mathrm{CH}$ often present with malocclusion and mandibular asymmetry (deviation of the chin to the unaffected side). $\mathrm{CH}$ is a self-limiting process that can cease active growth at any time, generally between the ages of 11 and 30 (4).

Bone scans can highlight areas of increased osteoblastic activity, aiding in the diagnosis of active or inactive $\mathrm{CH}$. This can guide treatment decisions: partial condylectomy for active $\mathrm{CH}$ or definitive operations for inactive $\mathrm{CH}$. Definitive interventions may involve osteotomies to correct any residual malocclusion or facial asymmetry. Postsurgical deformity may develop if osteotomies are performed while the $\mathrm{CH}$ is in an active phase (1).

Consequently, accurate assessment of condylar growth activity is crucial to guiding treatment options. Assessment

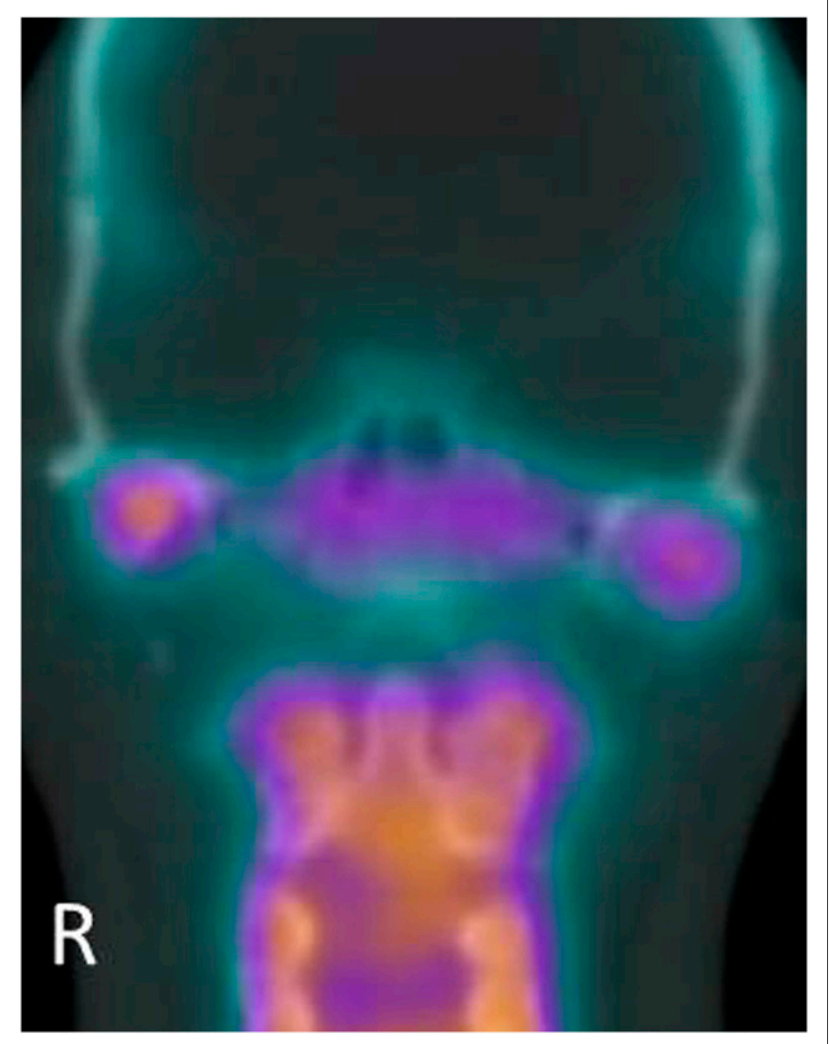

FIGURE 3. Coronal fusion image of SPECT and CT shows asymmetric uptake in bilateral mandibular condyles with increased uptake on right side compared with left side.

and diagnosis may be difficult if limited to planar imaging methods. SPECT methods are more sensitive and accurate in detecting growth activity and offer a quantitative means to determine growth differences between the left and right

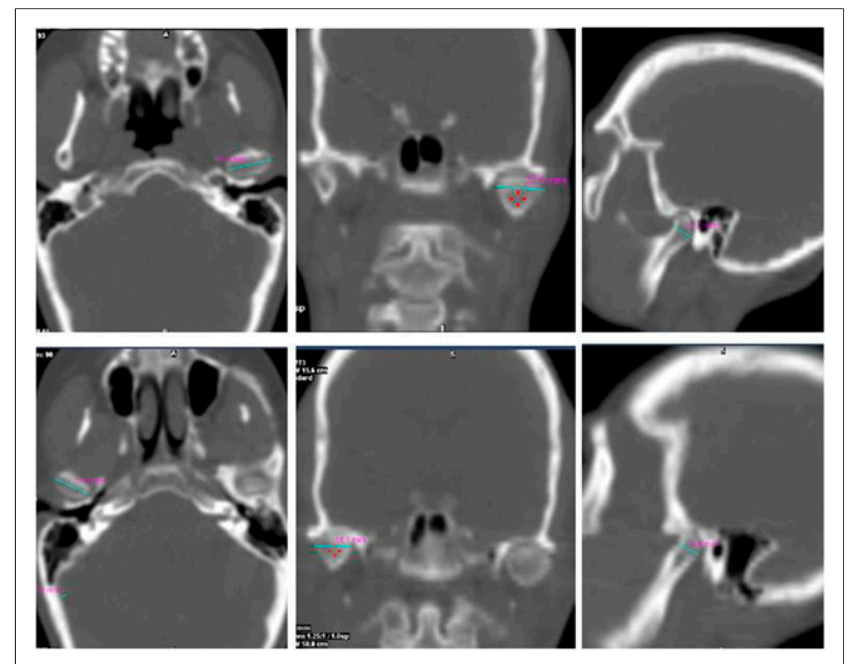

FIGURE 4. Low-dose CT shows left condyle (top row) as larger than right condyle (bottom row). Left condyle measures $21.4 \times 22.5 \times 12.2 \mathrm{~mm}$, and right condyle measures $17.8 \times 16.1 \times$ $9.8 \mathrm{~mm}$ in axial, coronal, and sagittal reconstruction images, respectively. 


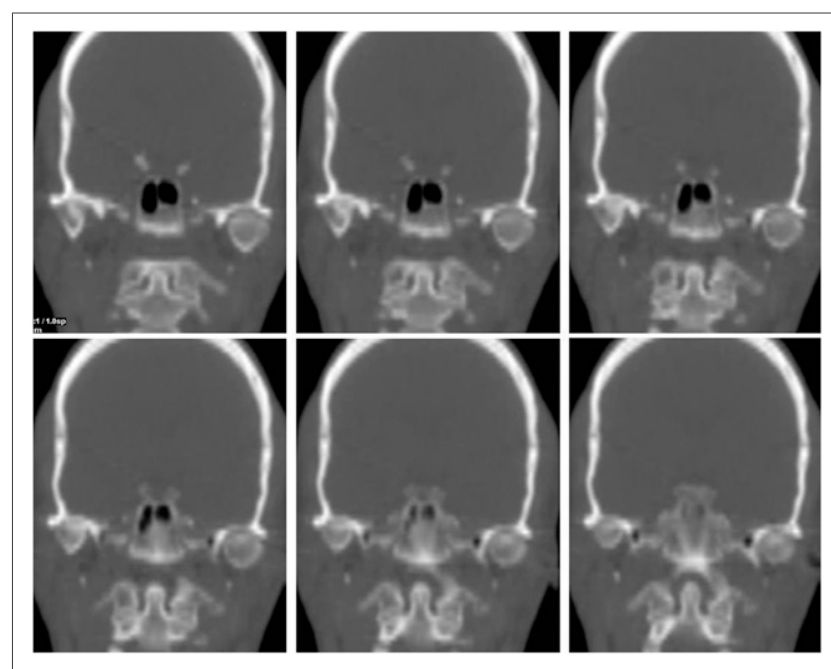

FIGURE 5. Serial low-dose CT coronal images show narrowed TMJ spaces with sclerosis that are worse on right side. CT scan was obtained immediately after SPECT scan using 4-slice, low-dose, spiral CT system, at $120 \mathrm{kV}$ and $20 \mathrm{~mA}$, with pitch of 1.75 . CT slices were reconstructed at 2.5-mm thickness using standard kernel filter. Radiation dose of this low-dose CT is about $2.7 \mathrm{mSv}$.

condyles. A $10 \%$ or more difference in the radionuclide uptake between the left and right condyles with increased uptake ipsilateral to the $\mathrm{CH}$ is considered to be evidence of active, growing $\mathrm{CH}(1-3)$.

Normally, the condyles are nearly symmetric and CT is a useful tool to measure and evaluate condylar dimensions (5).

Degenerative or inflammatory arthropathies of TMJs may complicate evaluation of growth activity as these conditions manifest with inherently increased ${ }^{99 \mathrm{~m} T c-M D P}$ uptake. However, this is generally not problematic as patients present with $\mathrm{CH}$ when they are relatively young $(1)$.
Degenerative bony changes occur more often and are more severe on the unaffected side than on the affected, hyperplastic side (4). The CT portion of SPECT/CT can be used to evaluate the underlying bony changes to confirm degenerative changes and rule out other possible causes of increased uptake, such as a tumor or trauma.

\section{CONCLUSION}

This case suggests that bone scan SPECT/CT provides an accurate means for the assessment of growth activity and detailed underlying anatomic information in a patient with $\mathrm{CH}$. These are helpful not only for diagnosis but also for the choice of an appropriate treatment. Degenerative arthropathies of TMJs may complicate evaluation of comparative uptake, but these conditions are more common and more severe on the side contralateral to the $\mathrm{CH}$.

\section{DISCLOSURE}

No potential conflict of interest relevant to this article was reported.

\section{REFERENCES}

1. Hodder SC, Rees JI, Oliver TB, Facey PE, Sugar AW. SPECT bone scintigraphy in the diagnosis and management of mandibular condylar hyperplasia. Br J Oral Maxillofac Surg. 2000;38:87-93.

2. Alyamani A, Abuzinada S. Management of patients with condylar hyperplasia: a diverse experience with 18 patients. Ann Maxillofac Surg. 2012;2: 17-23.

3. Wen B, Shen Y, Wang CY. Clinical value of ${ }^{99} \mathrm{Tc}^{\mathrm{m}}$-MDP SPECT bone scintigraphy in the diagnosis of unilateral condylar hyperplasia. ScientificWorldJournal. 20142014:1256256.

4. Nah KS. Hyperplastic conditions of the mandibular condyles. Korean J Oral Maxillofac Radiol. 2003;33:207-209.

5. Neto JV, Estrela C, Bueno MR, et al. Mandibular condyle dimensional changes in subjects from 3 to 20 years of age using cone-beam computed tomography: a preliminary study. Dental Press J Orthod. 2010;15:172-181.

\section{Erratum}

In Appendix A of the article "SNMMI and EANM Practice Guideline for Meckel Diverticulum Scintigraphy 2.0," by Spottswood et al. (J Nucl Med Technol. 2014;42:163-169), the pretreatment oral dose of ranitidine in adults referred for a Meckel scan was incorrectly stated to be $150 \mathrm{mg} / \mathrm{kg}$. The correct dose is $150 \mathrm{mg}$. The authors regret the error. 